\title{
Context-specific morphine withdrawal: Evidence that rearing reflects withdrawal, not exploration
}

\author{
JULIAN L. AZORLOSA and EVETTE L. SIMMONS \\ Southeastern Louisiana University, Hammond, Louisiana
}

\begin{abstract}
Several studies have demonstrated that contextual cues previously paired with morphine elicit conditioned withdrawal in the absence of the drug. Conditioned withdrawal signs have included circling, jumping, rearing, and wet dog shakes. One of these signs, rearing, may in fact reflect exploration rather than withdrawal. In those studies, rats had morphine paired or unpaired with distinctive contextual cues for several sessions and then were tested in that environment. During conditioning, the rats that received the drug in that context may have explored it less because they were sedated by the morphine. In the withdrawal test, this environment would have been more novel for these rats, as compared with the rats for which the contextual cues had been paired with saline. In the present study, rats were given eight injections of morphine, spaced $48 \mathrm{~h}$ apart, either paired or unpaired with distinctive contextual cues. During these sessions, the rats were physically restrained. Although both groups were equated for exploration of this context, the paired group, which received morphine in this context, displayed more rearing during the test session. These results suggest that rearing is an index of conditioned withdrawal.
\end{abstract}

Repeated administration of a drug can result in tolerance, defined by diminished effectiveness of the drug after multiple exposures. Repeated administration can also result in dependence, which is revealed by the appearance of withdrawal signs when the drug is discontinued. Siegel $(1975,1989)$ proposed a classical conditioning model of drug tolerance and dependence, in which the drug is the unconditioned stimulus (US) and the drug effects are the unconditioned responses (URs). Environmental (contextual) cues present during drug administration become conditioned stimuli (CSs), which acquire the capacity to elicit compensatory conditioned responses (CRs). These compensatory CRs serve a homeostatic function by reducing the drug URs, resulting in conditioned tolerance. In the absence of the drug, these compensatory CRs elicited by the CS constitute withdrawal. Current conceptualizations of this conditioning process now view URs not as drug effects, but rather as homeostatic reactions to the drug (e.g., Ramsey \& Woods, 1997; Siegel \& Allan, 1998). However, compensatory CRs still mediate tolerance and withdrawal.

Since Siegel (1975) proposed the classical conditioning model, there have been numerous studies confirming that

This study was funded by a Faculty Development grant from Southeastern Louisiana University to J.L.A. The morphine sulfate was a generous gift from the National Institute on Drug Abuse. The authors thank Shepard Siegel for his helpful comments on an earlier draft of this paper. Correspondence concerning this article should be addressed to J. L. Azorlosa, Center for Scientific Review, 6701 Rockledge Dr., Room 3190, MSC-7848, Bethesda, MD 20892 (e-mail: azorlosj@, csr.nih.gov). tolerance to several drugs, including ethanol, barbiturates, and opiates, is mediated, at least in part, by classical conditioning (see Goudie \& Demellweek, 1986; Siegel, 1989 , for reviews). Although contextual control of tolerance is a well-established phenomenon, control of drug withdrawal by contextual cues previously paired with the drug has received much less attention.

Kelsey and colleagues (Falls \& Kelsey, 1989; Kelsey, Aranow, \& Matthews, 1990) demonstrated contextual control of opiate withdrawal. In both studies, rats were given several sessions in which one group (paired) received morphine injections in a distinctive environment (DE) and saline injections in the home cage (HC). A second group (unpaired) received morphine in the $\mathrm{HC}$ and saline in the DE. A third group received saline in both locations. After these conditioning sessions, the rats were observed for opiate withdrawal signs in the DE following a saline injection. Consistent with Siegel's model, the paired group, as compared with the unpaired and saline groups, displayed more rears and jumps, which are considered to be signs of abstinent opiate withdrawal in rats (see, e.g., Linesman, 1977). In our laboratory, using the paired/unpaired design and different doses of morphine, we have found contextual control of rearing, jumping, circling, and wet dog shakes (Azorlosa, Hartley, \& Deffner-Rappold, 1994; Deffner-Rappold, Azorlosa, \& Baker, 1996). However, wet dog shakes were only demonstrated with a high dose $(75 \mathrm{mg} / \mathrm{kg})$. When a lower dose $(10 \mathrm{mg} / \mathrm{kg})$ was used, only signs indicating hyperactivity (rears, jumps, and circles) were observed. Generally, rearing was the most consistently observed sign. 
Recently, McDonald and Siegel (1998) hypothesized that the increased rearing in the paired groups, observed in those studies, might have been exploratory behavior and not conditioned withdrawal. In the paired/unpaired design used in those studies, the paired groups received conditioning sessions consisting of morphine in the DE and saline in the $\mathrm{HC}$. The unpaired groups received saline in the DE and morphine in the HC. McDonald and Siegel suggested that, during conditioning, the paired groups, sedated by morphine, explored the DE less than did the unpaired groups, which were not sedated. During testing in the DE with saline, the increased rearing in the paired groups was due to the relative novelty of that environment, as compared with the unpaired groups that had previously been exposed to it in an undrugged state (see, e.g., Scoles \& Siegel, 1986). Rather than indicating conditioned withdrawal, the increased rearing in the paired groups in those studies might simply have been an artifact of the paired/unpaired design. To test this alternative interpretation of rearing, McDonald and Siegel conditioned three groups of rats (paired, unpaired, and saline), using the paired/unpaired design described above. In addition, two other groups received sessions in a third environment. One group (MN) received morphine in this context and saline in the $\mathrm{HC}$. The other group $(\mathrm{SN})$ received saline in both locations. These two groups were not exposed to the DE during conditioning. Following 12 conditioning sessions, all the groups were tested with saline in the DE. Consistent with previous research, the paired group reared more than both the unpaired and the saline groups. However, groups $\mathrm{MN}$ and $\mathrm{SN}$, which had been trained in the third environment, also reared more than both the unpaired and the saline groups. In addition, $\mathrm{MN}$ reared as much as the paired group. Because groups MN and SN had never been in the DE prior to testing, the increased rearing was attributed to the novelty of the test environment. McDonald and Siegel suggested that these results render problematic previous interpretations of rearing as conditioned withdrawal.

The finding that group MN reared as much as the paired group raises some questions about rearing as a withdrawal measure. It does not, however, force the conclusion that the two groups exhibited increased rearing for the same reason. It is possible that the paired group reared because of conditioned withdrawal and that the MN group was exploring. Nonetheless, the results of McDonald and Siegel (1998) are provocative and deserve further attention. Perhaps, as those authors suggested, the sedative effect of morphine acted as a pharmacological restraint, which reduced exploration by the paired rats during training sessions. One way to explore this issue is to simulate the sedating effect of morphine by physically restraining both the paired and the unpaired groups during the conditioning sessions in the $\mathrm{DE}$, thus equating them for exploration. Any differences in rearing during the test could not be attributed to different levels of exploration during conditioning. This was the purpose of the present experiment.

\section{METHOD}

\section{Subjects}

The subjects were 16 experimentally naive male Sprague-Dawley rats weighing between 280 and $315 \mathrm{~g}$ at the start of the study. They were individually housed in the colony room and allowed ad-lib access to food and water. The colony room was lighted on a 14:10-h light:dark cycle.

\section{Drugs}

Morphine sulfate $(10 \mathrm{mg} / \mathrm{kg})$ was administered in a saline vehicle of $0.85 \% \mathrm{NaCl}$ in a volume of $1 \mathrm{ml} / \mathrm{kg}$. All injections were administered subcutaneously to the dorsal neck area.

\section{Distinctive Environment}

The DE consisted of two rooms, each containing four aluminum cages with black and white stripes on the sides. The cages were $23 \mathrm{~cm}$ wide, $28 \mathrm{~cm}$ high, and $35.5 \mathrm{~cm}$ long. White noise $(60 \mathrm{~dB}$, scale C) masked extraneous sounds. Desk lamps with $25-\mathrm{W}$ red light bulbs were mounted above each cage and provided the only illumination. The cages were cleaned with a pine-scented disinfectant between sessions.

\section{Procedure}

The rats were habituated to handling, injections, and tube restraints (IITC Inc., Model 81) during a 24-day period. Every other day, the rats received one habituation session in the morning and one in the afternoon. In each session, the animals received a saline injection, followed by placement in the tube restraints for $1 \mathrm{~h}$. On alternate days, the rats were handled. The purpose of these sessions was to minimize the chance that the restraint procedure would overshadow the DE during conditioning.

The conditioning trials began $48 \mathrm{~h}$ after the last habituation day. The 16 rats were randomly assigned to the paired or the unpaired groups. The paired rats received morphine in the DE and saline in the $\mathrm{HC}$. The unpaired rats received saline in the $\mathrm{DE}$ and morphine in the HC. The rats were weighed in the morning and then placed in the $\mathrm{DE}$ for $10 \mathrm{~min}$. After $10 \mathrm{~min}$, they received the appropriate injection and were placed in the tube restraints in the assigned cage in the DE. Cage assignment was counterbalanced between groups. One hour later, they were taken back to their HCs in the colony room. Five hours later, all the rats were given the appropriate injection and placed in the tube restraints on the table in the colony room. They remained there for $1 \mathrm{~h}$ and were then returned to the HC. The purpose of the restraint procedure in the colony room was to minimize the possibility of any associations between the restraint tubes and either morphine or saline injections or the DE. A total of eight conditioning trials were conducted, spaced $48 \mathrm{~h}$ apart.

On test day, $48 \mathrm{~h}$ after the last conditioning trial, the groups were tested for rearing. All the animals were placed in the DE cages and videotaped for $10 \mathrm{~min}$. An observer, blind to group assignment, viewed the tapes and recorded the frequency of rears (standing on hind paws, stretching torso upward), jumps (all four paws off floor), circles (continuous rotation), and wet dog shakes (brief shaking of head and body).

\section{RESULTS AND DISCUSSION}

One rat in the paired group became ill and died. A second, apparently healthy rat from the unpaired group died during the third conditioning session after the colony room morphine injection while restrained. In the first three sessions, several other animals appeared to have respiratory distress when drugged and restrained. On the fourth and fifth conditioning sessions, the morphine dose 
50 -

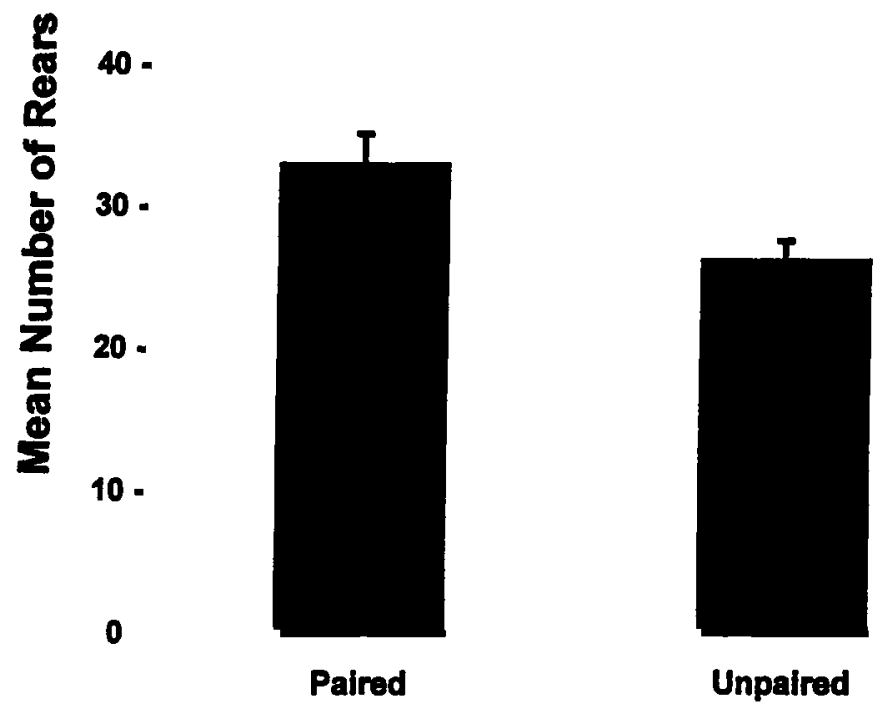

Figure 1. Mean number of rears during the 10 -min withdrawal test. Bars indicate standard errors of the mean.

was reduced to $5 \mathrm{mg} / \mathrm{kg}$. On sessions $6-8$, the dose was returned to $10 \mathrm{mg} / \mathrm{kg}$.

Mean number of rears is presented in Figure 1. The rats in the paired group reared more than those in the unpaired group, and this difference was statistically reliable $[t(12)=$ $2.68, p<.05]$. Figure 2 shows that the paired group also jumped more than the unpaired group $[t(12)=2.71, p<$ $.01]$. There were no differences between the groups for either circling or wet dog shakes.

The finding that the paired group reared and jumped more than the unpaired group is consistent with previous studies of conditioned withdrawal (Azorlosa et al., 1994;

2

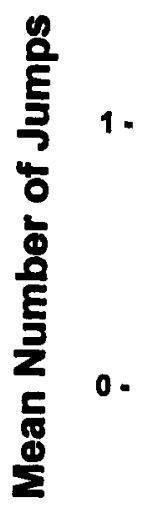

$-1$

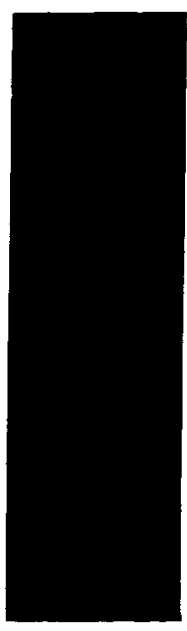

Paired

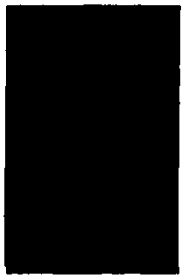

Unpaired

Figure 2. Mean number of jumps during the 10-min withdrawal test. Bars indicate standard errors of the mean. 
Deffner-Rappold et al., 1996; Falls \& Kelsey, 1989; Kelsey et al., 1990) and is consistent with the classical conditioning model of drug dependence, in which contextual cues, previously paired with a drug, elicit withdrawal signs (Siegel, 1989). The failure to observe conditioned wet dog shakes is also consistent with Deffner-Rappold et al., in which this sign was found with a much higher dose of morphine $(75 \mathrm{mg} / \mathrm{kg}$ ) but not with $10 \mathrm{mg} / \mathrm{kg}$, the dose used in the present study. These results also confirm that a relatively low dose of morphine is sufficient to demonstrate conditioned withdrawal.

The main finding of this study was that the paired group reared more than the unpaired group, despite the fact that both groups were restrained from any physical exploration after the injections. Before concluding that the rearing indicated conditioned withdrawal, two alternative explanations deserve discussion. First, increased rearing may have reflected sensitization rather than withdrawal. Previous studies (e.g., Shaham, Kelsey, \& Stewart, 1995) have shown that restraint-produced stress increases the locomotor effects of morphine. We attempted to control for these effects by habituating the rats to the restraint procedure prior to the conditioning sessions. This did not, of course, ensure that the restraint produced no stress during conditioning. Both groups were also restrained after both morphine and saline injections, to reduce any state-dependent effects or associations between restraint and morphine. However, this may not have eliminated all the potential confounds. Indeed, Shaham et al. also demonstrated that stress-induced sensitization could be conditioned. In their study, rats that received morphine immediately after being restrained became more active than rats that had the restraint procedure and morphine separated by $24 \mathrm{~h}$. On the basis of this study, it is conceivable that the behavioral sensitizing effects of morphine became conditioned to the DE in the paired rats and to the $\mathrm{HC}$ in the unpaired group, since that is where each group, respectively, was restrained and given morphine. However, this seems to be an unlikely explanation for the present results, because the increased activity observed by Shaham et al. was only found when the rats were tested with morphine. There were no differences when their groups, previously trained with morphine, were tested with saline, which was how the rats were tested in the present study.

Another interpretation is that the restraint manipulation did not entirely eliminate the effects of pharmacological restraint or state dependency suggested by McDonald and Siegel (1998). We attempted to control for differential pharmacological restraining effects by equating the groups for physical restraint. We attempted to control for state dependency effects by giving both groups 10 min of undrugged, unrestrained exploration of the DE at the beginning of each conditioning session and by testing them during this initial 10 -min period. Nonetheless, in a conditioning session, once the rats were injected and restrained, awareness of other cues (e.g., olfactory, auditory, or visual) may have been attenuated or altered in the paired rats, making the DE more novel for this group during testing. This explanation, although speculative, cannot be entirely discounted. However, the paired group also jumped more than the unpaired group. Jumping, to the best of our knowledge, is not confounded with exploration. Therefore, the most parsimonious explanation is that the increased rearing and jumping in the paired group reflected conditioned withdrawal.

The present results, however, do not allow us to rule out the suggestion of McDonald and Siegel (1998) that the rearing reported in previous studies (DeffnerRappold et al., 1996) was partially due to the relative novelty of the DE in the paired groups. The relative novelty could have been due to pharmacological restraint during conditioning, state dependency effects, or both. It is possible that the paired/unpaired design used in those studies produced rearing in the paired groups that was due to both conditioned withdrawal and increased exploration. Additional research is needed to address this issue.

\section{REFERENCES}

Azorlosa, J. L., Hartley, N. E., \& Deffner-Rappold, C. (1994). Context-specific morphine tolerance and withdrawal: The effects of interdose interval. Psychobiology, 22, 304-311.

Deffner-Rappold, C., Azorlosa, J. L., \& Baker, J. D. (1996). Acquisition and extinction of context-specific morphine withdrawal. Psychobiology, 24, 219-226.

Falls, W. A., \& Kelsey, J. E. (1989). Procedures that produce contextspecific tolerance to morphine also produce context-specific withdrawal. Behavioral Neuroscience, 103, 842-849.

Goudie, A. J., \& DEMELlweEk, C. (1986). Conditioning factors in drug tolerance. In S. R. Goldberg \& I. P. Stolerman (Eds.), Behavioral analysis of drug dependence (pp. 225-285). New York: Academic Press.

Kelsey, J. E., Aranow, J. S., \& Matthews, R. T. (1990). Contextspecific morphine withdrawal in rats: Duration and effects of clonidine. Behavioral Neuroscience, 104, 704-710.

LINESMAN, M. A. (1977). Naloxone-precipitated withdrawal as a function of the morphine-naloxone interval. Psychopharmacologia, 54, 159-164.

McDonald, R. V., \& Siegel, S. (1998). Environmental control of morphine withdrawal: Context specificity or stimulus novelty? Psychobiology, 26, 53-56.

RAMSEY, D. S., \& WoodS, S. C. (1997). Biological consequences of drug administration: Implications for acute and chronic tolerance. Psychological Review, 104, 170-193.

SCOLES, M. T., \& SiEgEL, S. (1986). The potential role of saline trials in morphine-induced place-preference conditioning. Pharmacology, Biochemistry \& Behavior, 25, 1169-1173.

Shaham, Y., Kelsey, J. E., \& Stewart, J. (1995). Temporal factors in the effect of restraint stress on morphine-induced behavioral sensitization in the rat. Psychopharmacology, 117, 102-109.

SIEGEL, S. (1975). Evidence from rats that morphine tolerance is a learned response. Journal of Comparative \& Physiological Psychology, 89, 498-506.

SIEGEL, S. (1989). Pharmacological conditioning and drug effects. In A. J. Goudie \& M. W. Emmett-Oglesby (Eds.), Psychoactive drugs (pp. 115-180). Clifton, NJ: Humana.

Siegel, S., \& Allan, L. G. (1998). Learning and homeostasis: Drug addiction and the McCollough effect. Psychological Bulletin, 124, 230-239.

(Manuscript received January 29, 1999; revision accepted for publication March 25, 1999.) 